\title{
SLC2A1 gene analysis of Japanese patients with glucose transporter 1 deficiency syndrome
}

\author{
Natsuko Hashimoto $^{1}$, Kuriko Kagitani-Shimono ${ }^{1,2}$, Norio Sakai ${ }^{1}$, Takanobu Otomo ${ }^{1}$, Koji Tominaga ${ }^{1,3}$, \\ Shin Nabatame ${ }^{1}$, Yukiko Mogami ${ }^{4}$, Yukitoshi Takahashi ${ }^{4}$, Katsumi Imai ${ }^{4}$, Keiko Yanagihara ${ }^{5}$, Takeshi Okinaga ${ }^{1}$, \\ Toshisaburo Nagai ${ }^{6}$, Masako Taniike ${ }^{2,3}$ and Keiichi Ozono ${ }^{1}$
}

Glucose transporter 1 deficiency syndrome (Glut1-DS) is a congenital metabolic disorder characterized by refractory seizures with early infantile onset, developmental delay, movement disorders and acquired microcephaly. Glut1-DS is caused by heterozygous abnormalities of the SLC2A1 (Glut1) gene, whose product acts to transport glucose into the brain across the blood-brain barrier. We analyzed the SLC2A1 gene in 12 Japanese Glut1-DS patients who were diagnosed by characteristic clinical symptoms and hypoglycorrhachia as follows: all patients had infantile-onset seizures and mild to severe developmental delay, and ataxia was detected in 11 patients. For the 12 patients, we identified seven different mutations (three missense, one nonsense, two frameshift and one splice-site) in exons and exon-intron boundaries of the SLC2A1 gene by direct sequencing, of which six were novel mutations. Of the remaining five patients who had no point mutations and underwent investigation by multiplex ligation-dependent probe amplification, a complex abnormality with deletion and duplication was identified in one patient: this is the first case of such recombination of the SLC2A1 gene. Changes in regulatory sequences in the promoter region or genes other than SLC2A1 might be responsible for onset of Glut1-DS in the other four patients (33\%) without SLC2A1 mutation.

Journal of Human Genetics (2011) 56, 846-851; doi:10.1038/jhg.2011.115; published online 20 October 2011

Keywords: glucose transporter 1 deficiency syndrome; mutation; SLC2A1

\section{INTRODUCTION}

Glucose transporter 1 deficiency syndrome (Glut1-DS: OMIM no. 606777) was first described by De Vivo et al. ${ }^{1}$ in 1991 as a disease with impaired glucose transport across the blood-brain barrier and characterized by hypoglycorrhachia, seizures and developmental delay. Validation of their observation was confirmed by the discovery that Glut1-DS was caused by genetic abnormalities of the SLC2A1 (Glut-1) gene (OMIM $\left.{ }^{\star} 138140\right)$ encoding Glut1, by which glucose transport across the blood-brain barrier is mediated. The SLC2A1 gene is located on the short arm of chromosome $1(1 \mathrm{p} 34.2)^{2}$ and is about $35 \mathrm{~kb}$ in length, containing 10 exons. $^{3}$ Almost 60 mutations including missense, nonsense, frameshift and splice-site mutations of the SLC2A1 gene have been reported to date in Glut1DS patients. ${ }^{4}$ In addition, chromosomal abnormalities, such as microdeletions including the SLC2A1 gene, have also been recently reported. 5,6

Here, we report the clinicogenetic characteristics of 12 Japanese patients with Glut1-DS. Initial mutation analysis was performed by direct sequencing, and multiplex ligation-dependent probe amplification (MLPA) was subsequently performed in the remaining patients in whom no point mutation was identified. This is the first report documenting clinicogenetic features in a series of Japanese patients with Glut1-DS.

\section{MATERIALS AND METHODS}

\section{Patients}

In all 13 Glut1-DS patients, who were diagnosed at Osaka University Hospital or whose blood sample had been sent for genetic analysis from other institutions from 2004 to 2011, were subjected to the study. Diagnosis was made according to clinical and laboratory features: infantile-onset seizures, developmental delay, EEG abnormalities aggravated by fasting and improved by food intake, low CSF/blood glucose ratio $(<0.5)$ after $4-5 \mathrm{~h}$ fasting despite low to normal CSF lactate concentration and normal levels of CSF cells and protein. 3-O-Methyl-D-Glucose (3-OMG) uptake by erythrocytes was measured according to previously reported methods. ${ }^{7}$ This study was exempted from full review and approved by the Osaka University Graduate School of Medicine Institutional Review Board. Written informed consent was obtained from the parents of all the children who were recruited to this study.

${ }^{1}$ Department of Pediatrics, Osaka University Graduate School of Medicine, Osaka, Japan; ${ }^{2}$ United Graduate School of Child Development, Osaka University Graduate School of Medicine, Osaka, Japan; ${ }^{3}$ Department of Molecular Research Center for Children's Mental Development, Osaka University Graduate School of Medicine, Osaka, Japan; ${ }^{4}$ National Epilepsy Center, Shizuoka Institute of Epilepsy and Neurological Disorders, Shizuoka, Japan; ${ }^{5}$ Osaka Medical Center and Research Institute for Maternal and Child Health, Osaka, Japan and ${ }^{6}$ Division of Health Sciences, Osaka University Graduate School of Medicine, Osaka, Japan

Correspondence: Dr K Kagitani-Shimono, Department of Pediatrics, Osaka University, Graduate School of Medicine, 2-2 Yamadaoka, Suita, Osaka 565-0871, Japan.

E-mail: kuriko@ped.med.osaka-u.ac.jp

Received 14 June 2011; revised and accepted 15 September 2011; published online 20 October 2011 


\section{Mutational analysis of SLC2A1}

Genomic DNA was isolated from heparinized blood using QuickGene DNA whole blood Kit S and Nucleic Acid Isolation System QuickGene-810 (FUJIFILM, Tokyo, Japan). PCR primer sets were designed to cover the exons and exon-intron junctions of the SLC2A1 gene. The primers used are shown in Table $1 .{ }^{8} \mathrm{PCR}$ was conducted in $20 \mu \mathrm{l}$ volume containing $50 \mathrm{ng}$ genomic DNA, $0.5 \mu \mathrm{m}$ each primer, $0.2 \mathrm{~mm}$ each dNTP and $1 \mathrm{U}$ Taq DNA polymerase (Takara Taq, Takara Bio Inc. Otsu Shiga, Japan). The PCR reaction used 35 cycles of amplification with denaturation at $95{ }^{\circ} \mathrm{C}$ for $4 \mathrm{~min}$, annealing for $30 \mathrm{~s}$ at $64^{\circ} \mathrm{C}$ for $1 \mathrm{~min}$, extension at $72^{\circ} \mathrm{C}$ for $2 \mathrm{~min}$, and followed by a final extension at $72{ }^{\circ} \mathrm{C}$ for $10 \mathrm{~min}$. The amplified products were purified with Takara SUPREC-PCR (Takara Bio Inc.). The SLC2A1 gene was directly sequenced using $20 \mathrm{ng}$ of purified DNA with BigDye Terminator vl.1 Cycle Sequencing Kit (Applied Biosystems, Foster, CA, USA). The pathogenetic nature of novel mutations was confirmed by their absence in 100 control subjects.

\section{MLPA analysis}

The principle for MLPA, which is a technique for measuring allele dosage by the hybridization of the complementary probes, has been described previously. ${ }^{9}$ The SALSA SLC2A1 region test reagent set (P138 probe mix; MRC-Holland, Amsterdam, The Netherlands) contains one probe for each exon of the SLC2A1 gene and 12 reference probes (www.mrc-holland.com). All runs included DNA from unrelated controls for calibration. MLPA was entrusted to FALCO biosystems (Kyoto, Japan).

\section{RESULTS}

\section{Clinical characteristics}

The clinical signs and laboratory data for the study patients are listed in Table 2. There were three boys and nine girls ages 6-15 years.

\section{Table 1 Primer set for the direct sequencing of the $S L C 2 A 1$ gene}

\begin{tabular}{lll} 
Exon & Forward primer & Reverse primer \\
\hline 1 & 5'-AACAGCGAGCGTGCCGGTCGCTAGT-3' & 5'-TAAGGCGGGCAGGAGTCTGCGCCTT-3' \\
2 & 5'-CTCCCAGACACGCCTATAACAGT-3' & 5'-GGCTGGTGTCCATAAGCCAACG-3' \\
$3-4$ & 5'-GCTTGCTCACCCAGGCTGCAT-3' & 5'-GTGCCAGGCAGGTAGATCCT-3' \\
$5-8$ & 5'-AAAGGGGGTCAGGGCAGAGGCGCTCA-3' & 5'-GCATCCCTCACTCTCCAGAACCT-3' \\
$9-10$ & 5'-AACTTTTCCCCCTCTCCGTCATC-3' & 5'-TGTGCTCCTGAGAGATCCTTA-3' \\
\hline
\end{tabular}

All patients showed early-onset (2-11 months of age) and refractory seizures. Seizure type was diverse and included generalized tonicclonic, atonic, absence and complex partial seizures. A total of 8 patients had microcephaly below -2 s.d. All of them had developmental delay to various degrees of severity. All except one had truncal ataxia and eight showed dystonia.

Laboratory examination revealed hypoglycorrhachia (CSF glucose $26-38 \mathrm{mg} \mathrm{dl}^{-1}$ and CSF/blood glucose ratio: $0.28-0.48$ ) in all patients. 3 -OMG uptake by erythrocytes was also decreased (to $47-56 \%$ of controls) in eight patients, but one patient (case 5) was within the normal range of erythrocyte 3-OMG uptake. The clinical features of two informative cases are detailed later in this section.

\section{SLC2A1 mutations on direct sequencing}

A summary of the results of the SLC2A1 gene analysis is shown in Table 3. Direct sequencing of the SLC2A1 gene revealed mutations in seven patients (cases 2, 5, 6, 7, 9, 10 and 11). Among them, three missense mutations (cases 5, 6 and 9) were noted. The mutation found in case 9 was the same as previously reported by Wang et al. ${ }^{9} \mathrm{~A}$ nonsense mutation was found in case 11 (c.1272 T>A, p.Y424X). Cases 7 and 11 showed frameshift mutations (case 7: c.707_708delAC, p.D236GfsX4 and case 11: c.431_432delTG, p.V144GfsX2). A splicesite mutation was found in case 2 (c.679+1G $>$ A). Collectively, direct sequencing of the gene revealed seven mutated alleles among 12 Glut1-DS patients, of which six were novel mutations. The novel missense mutation found in case 2, 5, 6, 7 and 11 was not found in 100 normal control subjects. The location for each mutation on the $S C L 2 A 1$ gene is indicated in Figure 1. No specific localization of mutations was found.

\section{Quantitative analysis by MLPA}

Further analysis using the MLPA method was performed in five patients in whom direct sequencing failed to disclose any mutation. In one patient (case 12), relative copy number was decreased from exon 1-8 and increased from exon 9-10. Although her father did not have any abnormalities, her mother had an increased relative copy

Table 2 Clinical characteristics of Glut1-DS patients

\begin{tabular}{|c|c|c|c|c|c|c|c|c|c|c|c|}
\hline \multirow[b]{2}{*}{$\begin{array}{l}\text { Patient } \\
\text { no. }\end{array}$} & \multirow[b]{2}{*}{ Gender } & \multirow[b]{2}{*}{$\begin{array}{l}\text { Age at } \\
\text { study (y) }\end{array}$} & \multicolumn{3}{|c|}{ Biochemical data } & \multicolumn{6}{|c|}{ Clinical signs } \\
\hline & & & $\begin{array}{c}\text { CSF/blood glucose } \\
\left(\mathrm{mgdl}^{-1}\right)\end{array}$ & $\begin{array}{c}\text { CSF/blood } \\
\text { glucose ratio }\end{array}$ & $\begin{array}{l}\text { Erythrocyte 3-OMG } \\
\text { uptake (\% normal) }\end{array}$ & $\begin{array}{c}\text { Seizure } \\
\text { onset (mo) }\end{array}$ & $\begin{array}{l}\text { Seizure } \\
\text { type }\end{array}$ & $\begin{array}{c}\text { Micro } \\
\text { cephalya }\end{array}$ & $\begin{array}{c}\text { Mental } \\
\text { impairment }^{\mathrm{b}}\end{array}$ & Ataxiac $^{\mathrm{c}}$ & Dystonia $^{\mathrm{d}}$ \\
\hline 1 & $\mathrm{~F}$ & 7 & $27 / 73$ & 0.36 & NA & 2 & GTC, CPS, Ato & + & +++ & +++ & + \\
\hline 2 & $\mathrm{~F}$ & 12 & $32 / 89$ & 0.39 & 56 & 2 & GTC, CPS & + & +++ & ++ & + \\
\hline 3 & $\mathrm{M}$ & 15 & $35 / 90$ & 0.39 & 48 & 2 & CPS, Abs & \pm & ++ & ++ & + \\
\hline 4 & $\mathrm{~F}$ & 12 & $26 / 83$ & 0.31 & 52 & 3 & GTC, CPS, Abs, Ato & + & +++ & +++ & + \\
\hline 5 & $M$ & 14 & $38 / 78$ & 0.48 & 102 & 3 & GTC,GT & + & +++ & +++ & - \\
\hline 6 & $\mathrm{~F}$ & 6 & $28 / 85$ & 0.32 & Decreased & 6 & GTC, Abs, CPS & - & ++ & ++ & + \\
\hline 7 & $\mathrm{~F}$ & 6 & $28 / 90$ & 0.32 & NA & 6 & CPS & - & + & + & + \\
\hline 8 & $\mathrm{~F}$ & 9 & $33 / 82$ & 0.4 & Decreased & 6 & Abs, Ato, CPS & + & + & + & - \\
\hline 9 & $\mathrm{~F}$ & 10 & $36 / 87$ & 0.41 & 56 & 8 & GTC, Abs & - & + & - & - \\
\hline 10 & M & 14 & $29 / 82$ & 0.35 & 47 & 9 & GTC, CPS & + & ++ & ++ & + \\
\hline 11 & $\mathrm{~F}$ & 4 & $26 / 90$ & 0.28 & NA & 9 & $\mathrm{GT}, \mathrm{Abs}$ & - & ++ & ++ & - \\
\hline 12 & $\mathrm{~F}$ & 15 & $30 / 84$ & 0.38 & 51 & 11 & CPS, Ato & + & +++ & ++ & + \\
\hline
\end{tabular}

Abbreviations: Abs, absence seizure; Ato, atonic seizure; CPS, complex partial seizure; CSF, cerebrospinal fluid; GT, generalized tonic seizure; GTC, generalized tonic-clonic seizure; mo, month;

NA, not assessed; 3-OMG, 3-O-methyl-D-glucose; y, year.

arade:,$- \leqslant 1$ s.d. (standard deviation); $\pm \leqslant 1.5$ s.d.;,$+ \leqslant 2$ s.d.

GGrade: -, none (DQ (developmental quotient) 70-100); +, mild (DQ 50-70); ++, moderate (DQ 35-50); +++, severe (DQ 20-35).

'Grade: -, none; +, mild; ++, moderate; +++, severe.

dGrade: - , absent; +, present. 
Table 3 Results of SLC2A1 gene analysis in Glut1-DS patients

\begin{tabular}{rlllll}
\hline Patient no. & Mutation & Location & Nucleotide change & Type of mutation & Family member analysis \\
\hline 1 & Not found & & & \\
2 & c.679+1G $>$ A & Intron 5 & c.679+1G $>$ A & Splice-site \\
3 & Not found & & & & \\
4 & Not found & & & Father (-) Mother (+) Brother (+) \\
5 & p.M344T & Exon 8 & c.1031T $>$ C & Missense & \\
6 & p.E41K & Exon 3 & c.121G $>$ A & Missense & \\
7 & p.D236GfsX4 & Exon 6 & c.707_708delAC & Frameshift & Father (-) Mother (-) Brother (-) \\
8 & Not found & & & & \\
9 & p.G130Sa & Exon 4 & c.388G $>$ A & Missense & \\
10 & p.Y424X & Exon 9 & c.1272T $>$ A & Nonsense & Frameshift \\
11 & p.V144GfsX2 & Exon 4 & c.431_432delTG & Deletion Duplication & Father (-) Mother (dup Ex9-10) \\
12 & del Ex1-8, dup Ex9-10 & Exon 1-10 & & &
\end{tabular}

autation previously reported in other patients. ${ }^{9}$

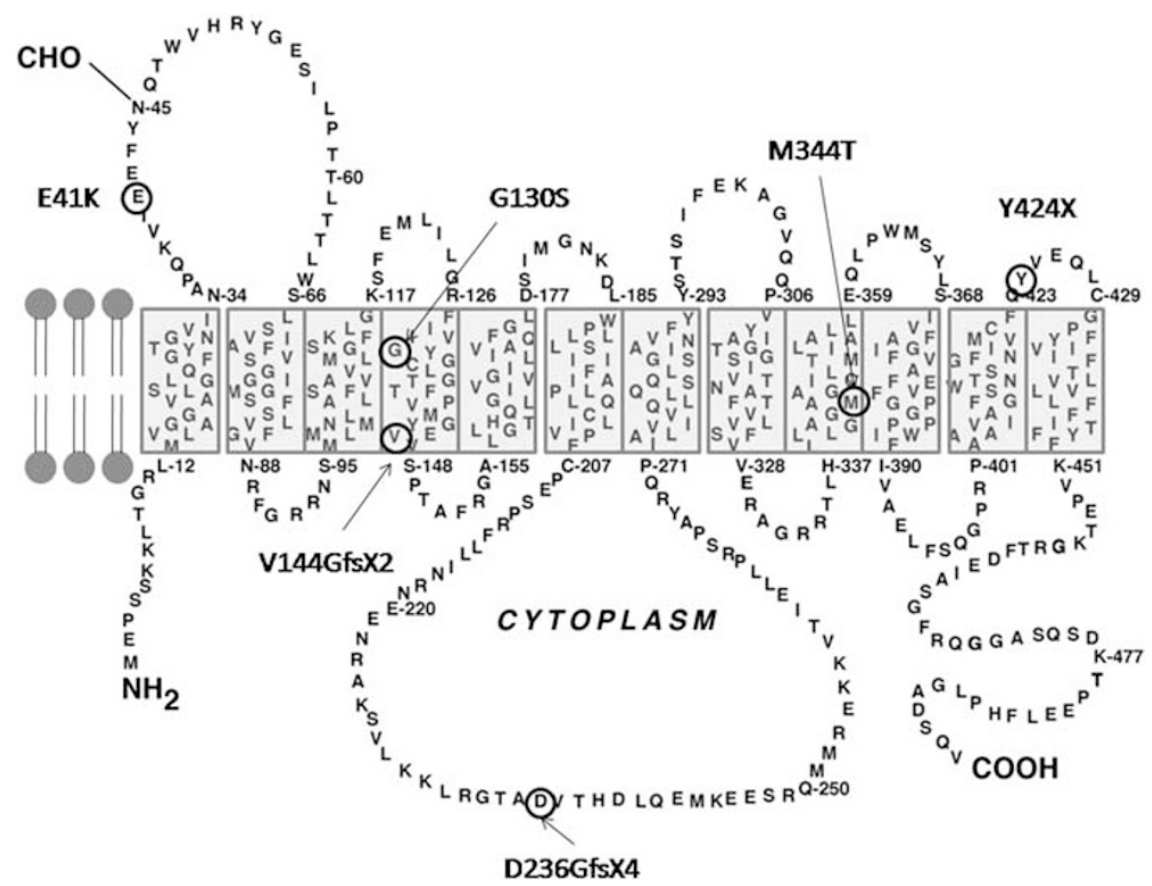

Figure 1 Location of six novel mutations (three missense, two frameshift and one nonsense) from Glut1-DS patients in the Glut1 protein transmembrane configuration. Mutations are scattered without a specific hotspot.

number between exon 9-10. This suggests that her mother originally had duplication in exon $9-10$ as a polymorphism and that further chromosomal recombination in front of the duplication occurred, leading to the onset of Glut1-DS in the proband (Figure 2).

\section{Case 5 presentation}

This 14-year-old male was the first child born to non-consanguineous parents. He was born at 37 gestational weeks (birth weight $2410 \mathrm{~g}$ ) without asphyxia by normal delivery. He developed tonic seizures at 3 months of age and his seizures were frequent and refractory to 10 anti-epileptic drugs including adrenocorticotropic hormone. His cognitive impairment was severe and he has never learned a single word. He manifested hypotonia, increased tendon reflex and ataxia but no dystonic posture. At the age of 9 years, he was suspected to be suffering from Glut1-DS because of low CSF glucose concentration
(Table 1), although glucose uptake by erythrocytes was not decreased. Direct sequencing analysis revealed a novel missense mutation (c.1031T > C, p.M344T).

A younger brother of the proband was also born by normal delivery. At 4 years of age, he developed monthly tonic-clonic seizures while in a fasting state before breakfast. He showed neither ataxia nor dystonia on fasting. His CSF glucose was slightly low (CSF/blood glucose; $41 / 71 \mathrm{mg} \mathrm{dl}^{-1}$ ). Although he did not show any motor disability, his cognitive function was mildly impaired. He has the same mutation as his brother.

When screened, the mother and a brother were also found to have the relevant mutation of the SLC2A1 gene but not their father (Figure 3). The mother's history revealed one episode of seizures at 6 years of age. The two brothers of the mother had been diagnosed with schizophrenia and autism, respectively, but we were unable to gain 
a

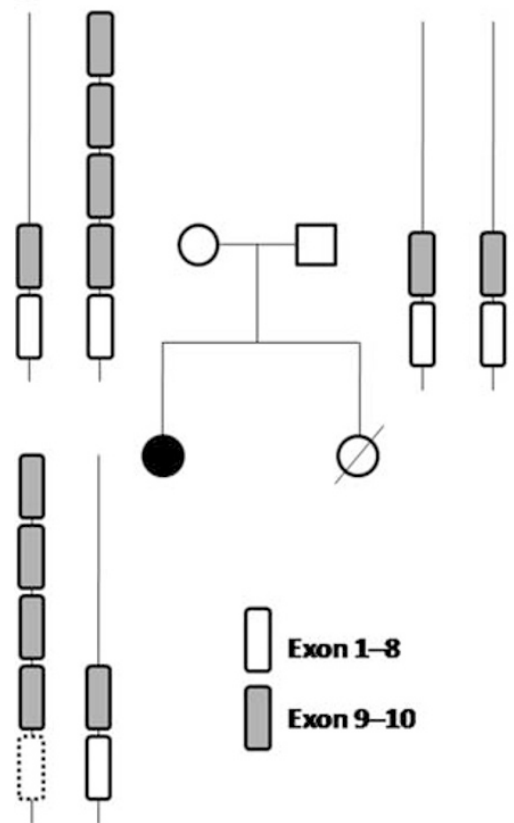

b
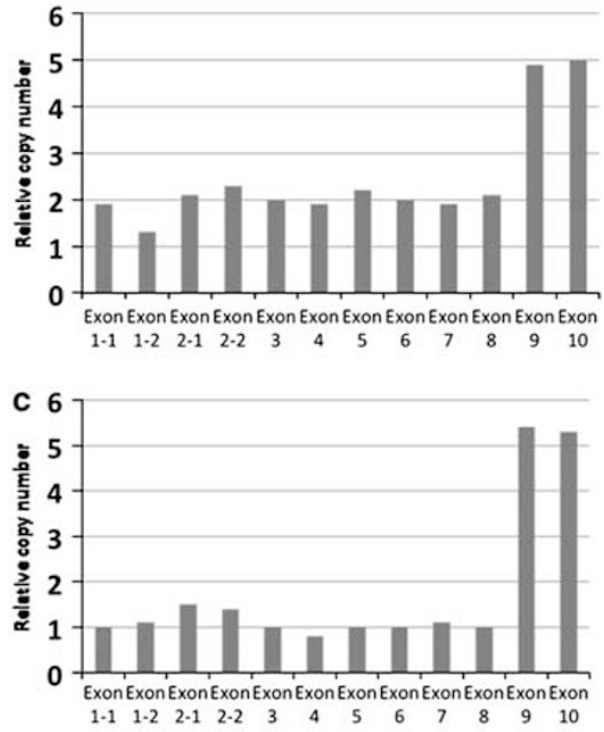

Figure 2 Family pedigree (a) and MLPA results in case 12. Although a relative copy number of around two was observed for normal controls, the mother of the proband had a copy number of about five in exon 9-10 (b). In case 12, there is a copy number of around one from exon 1-8 and about five from exon 9-10 (c). This means that case 12 has de novo microdeletion in exon 1-8 and duplication in exon 9-10. The latter is not pathogenetic as it is shared with her asymptomatic mother.

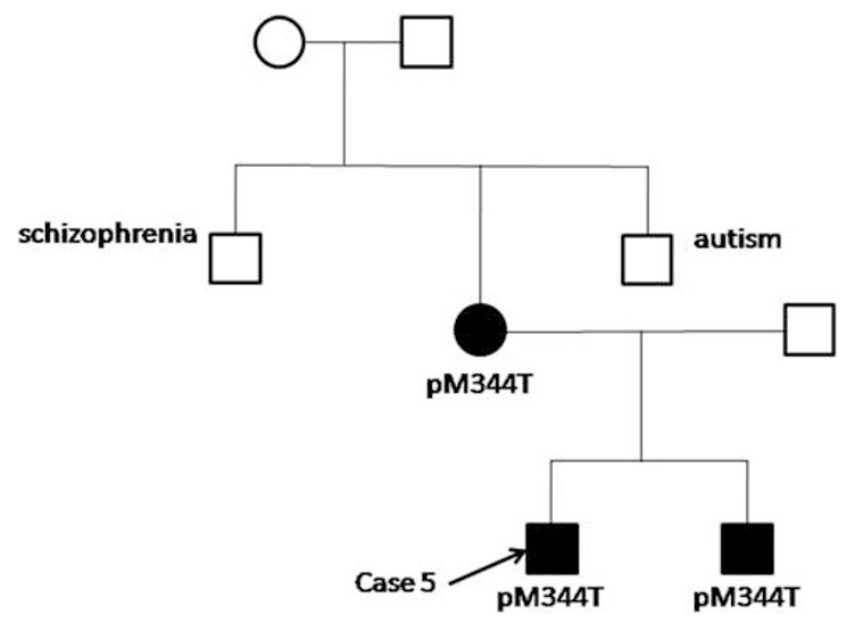

Figure 3 Family pedigree of case 5 . The proband, his brother and mother had same mutation. His father did not have the mutation. The mother's older brother was diagnosed with schizophrenia in his fourth decade of life and her younger brother was diagnosed with autism and was institutionalized in a shelter for intellectually disabled people.

consent for their SLC2A1 gene analysis. The pathogenetic nature of the mutation was confirmed by its absence in his father and one hundred non-consanguineous normal control subjects.

\section{Case 12 presentation}

This 15-year-old female was the first-born child of healthy nonconsanguineous parents. She was born after full-term pregnancy by vaginal delivery with no complication. Her birth weight was $2836 \mathrm{~g}$. She developed truncal ataxia and hypotonia at 6 months of age. At 11 months of age, she developed hemiconvulsions and atonic seizures just before breakfast. Phenobarbital was started and her seizures were well controlled. When phenobarbital was withdrawn at 5 years of age, atonic seizures recurred on fasting. Although she started to speak words at 15 months of age, her intellectual delay became severe thereafter. At 8 years of age, she was diagnosed with Glut-1 DS on the basis of clinical features as well as hypoglycorrhachia and decreased 3-OMG uptake by erythrocytes (Table 1).

At the age of 12 years old, she was admitted to our hospital to start a ketogenic diet. She had microcephaly (head circumference $50.8 \mathrm{~cm}$ ) but no other facial dysmorphia. Although she could walk without support, she easily fell to the ground and was unable to walk for a long distance because of spastic gait and ataxia. She could have a simple conversation, her intelligence quotient was low at 32 and she had dysarthria. MPLA analysis revealed duplication in exon 9-10, deletion in exon 1-8 and duplication in exon 9-10. Her mother possessed the same duplication in exon 9-10 without any neurological symptoms.

\section{DISCUSSION}

Classic Glut1-DS is characterized by early-onset epilepsy, developmental delay and acquired microcephaly, which is an aspect of encephalopathy. Klepper et al. ${ }^{10}$ proposed diagnostic criteria for Glut1-DS as follows: seizures, developmental delay, complex movement disorder and fasting EEG changes improving postprandially. Original laboratory criteria for Glut1-DS consisted of hypoglycorrhachia $\left(<40 \mathrm{mg} \mathrm{dl}^{-1}\right)$, low CSF/blood glucose ratio $(<0.4)$ and reduced erythrocyte glucose uptake (uptake cutoff point at $60 \%$ uptake). ${ }^{6}$ After the discovery of $S L C 2 A 1$ as a gene responsible for Glut1-DS, many mutations have been recognized that show autosomal dominant inheritance. However, as more cases with documented gene abnormalities of the SLC2A1 gene have been reported, atypical cases have come into light such as a case of movement disorder without 
epilepsy $^{11}$ and another with autosomal recessive inheritance. ${ }^{12}$ Furthermore, it was recognized that a wide clinical syndromes existed resulting from different SLC2A1 gene mutations. For example, Glut1DS was reported to occur in $>10 \%$ of patients with early-onset absence epilepsy and some of them expressed mild manifestations. ${ }^{13}$ Furthermore, some patients with SLC2A1 mutations have been reported to have milder hypoglycorrhacia $\left(40-52 \mathrm{mg} \mathrm{dl}^{-1}\right)$ and relatively high CSF/blood glucose ratio $(0.45-0.57)$ as compared with original Glut1-DS criteria. However, no Glut1-DS patients showed normal CSF glucose concentration. ${ }^{14}$ On the other hand, Glut1-DS cases have been reported which had normal erythrocyte glucose uptake. ${ }^{15}$ Therefore, in order not to overlook milder or atypical cases of Glut1-DS, usage of a broader criteria including mild hypoglycorrhacia or normal erythrocyte glucose uptake may be useful. These varied clinical and laboratory findings indicate the increasing importance of gene testing in the diagnosis of the patients suspected of having Glut1-DS. We report SLC2A1 gene analysis in 12 Japanese patients who were diagnosed on the basis of clinical features including early-onset epilepsy and hypoglycorrhachia. This is the first report of a large number of Japanese patients with Glut1-DS being subject to genetic analysis.

We analyzed genomic abnormalities for SLC2A1 including the case with typical clinical pictures and hypoglycorrhacia regardless of erythrocyte glucose uptake and found eight abnormalities including seven mutations, of which six were novel. The locations of each mutation in the transmembrane configuration of the Glut1 protein are shown in Figure 1. Although several hot spots for recurrent mutations have been previously reported, as many as 60 mutations have been reported scattered in transmembrane, intracellular and extracellular domains. ${ }^{16}$

We made sure that they didn't match the reported polymorphism in the database (Japanese Single-Nucleotide Polymorphisms), ${ }^{17}$ and also confirmed that the mutations were not present in one hundred healthy controls. All mutations caused the change of amino acid in SLC2A1. The nonsense and frameshift mutations are highly likely to induce the reduced function of Glut 1 . In case of the missense mutation, two patients out of three (cases 6 and 9) with missense mutations showed evidence of abnormal Glut 1 function supported by reduced 3-OMG uptake. The pathogenicity of the missense mutation in the remaining one patient (case 5) was unfortunately unsupported by reduced 3OMG uptake. Recently Glut1-DS patients with T295M mutation have been reported to have normal 3-OMG uptake in erythrocytes. However, in functional studies, Glut1 conformational change asymmetrically affects the efflux of glucose from cells as compared with its influx. ${ }^{15}$ Thus, influx measured by erythrocyte glucose uptake can be normal. ${ }^{18}$ Although we did not measure either glucose efflux or conformational change, we can speculate that this kind of mechanism could be associated with case 5 .

In addition, we revealed the first case with a complex rearrangement of deletion and duplication using the novel MLPA method (case 12). In this patient, deletion of exon 1-8 was the pathophysiological recombination, because her mother with same duplication did not have any symptoms and Glut1-DS is a congenital metabolic disorder induced by haploinsufficiency of the SLC2A1 gene. ${ }^{12}$

With respect to the genotype-phenotype correlation, we could not find a definite relationship between clinical severity indicated by seizure onset and/or CSF glucose concentration, CSF/blood glucose ratio and 3-OMG uptake. For example, case 5 had relatively mild hypoglycorrhachia, he had the same SLC2A1 mutation as his brother and mother, which indicates they were familial cases with an autosomal dominant trait. The proband case 5 manifested symptoms of 'encephalopathy' with refractory seizures and severe developmental delay. Despite his brother having the same CSF glucose concentration as that of the proband, he had considerably milder symptoms. The description of mutation-positive family members of case 5 agrees with previous reports documenting highly variable clinical symptoms in a Glut1-DS family, ranging from no clinical manifestation at all to the severe classic phenotype. ${ }^{13}$

Regarding seizure type, the predominant seizure types were generalized tonic-clonic seizure and complex partial seizures followed by absence and atonic seizures. Glut1-DS would appear to be a syndrome with variable generalized epilepsy. It is possible that most of our cases harbored classic and relatively severe phenotypes, which probably limited the ability to detect any correlation between genotype and phenotype.

Finally, no SLC2A1 mutation gene was found in five or our 12 patients $(33.3 \%)$ despite the presence of hypoglycorrhachia and typical clinical symptoms of Glut1-DS. Leen et al. ${ }^{16}$ reported that $59 \%$ of 132 Glut1-DS patients were negative on genetic analysis for SLC2A1 mutation. They suspected that regulatory sequences of the SLC2A1 gene such as promoter sequences and/or sequences deep within introns may have been underdiagnosed. In our series, there were no clinical differences between mutation-positive and -negative cases. Thus, mutation in regulatory sequences and/or other modulating genes could well affect Glut1 activity. Furthermore, loss of activity of hexokinase I, which involve glucose transport in the endothelial cells, may contribute to pathophysiology in these cases. ${ }^{19}$ In conclusion, as it is now possible to improve the prognosis of Glut1-DS with a ketogenic diet, it is important to make a definite diagnosis as early as possible. As there is a wide range of clinical manifestations and falsenegative patients when using the original criteria for Glut1-DS diagnosis, it is important to identify genetic abnormalities not only by the direct sequencing but also by MLPA. Further studies are needed for the detection of potentially secondary or modifier genes.

\section{ACKNOWLEDGEMENTS}

This work was supported by Eisai (project no. J109001037) and UCB Japan (project no. J099001110).

1 De Vivo, D. C., Trifiletti, R. R., Jacobson, R. I., Ronen, G. M., Behmand, R. A. \& Harik, S. I. Defective glucose transport across the blood-brain barrier as a cause of persistent hypoglycorrhachia, seizures, and developmental delay. N Engl J Med. 325, 703-709 (1991).

2 Shows, T. B., Eddy, R. L., Byers, M. G., Fukushima, Y., Dehaven, C. R., Murray, J. C. et al. Polymorphic human glucose transporter gene (GLUT) is on chromosome 1 p31.3p35. Diabetes. 36, 546-549 (1987).

3 Fukumoto, H., Seino, S., Imura, H., Seino, Y. \& Bell, G. I. Characterization and expression of human HepG2/erythrocyte glucose-transporter gene. Diabetes. 37, 657-661 (1988).

4 Seidner, G., Alvarez, M. G., Yeh, J. I., O'Driscoll, K. R., Klepper, J., Stump, T. S. et al. GLUT-1 deficiency syndrome caused by haploinsufficiency of the blood-brain barrier hexose carrier. Nat Genet. 18, 188-191 (1998).

5 Levy, B., Wang, D., Ullner, P. M., Engelstad, K., Yang, H., Nahum, O. et al. Uncovering microdeletions in patients with severe Glut-1 deficiency syndrome using SNP oligonucleotide microarray analysis. Mol. Genet. Metab. 100, 129-135 (2010).

6 Klepper, J., Garcia-Alvarez, M., O'Driscoll, K. R., Parides, M. K., Wang, D., Ho, Y. Y. et al. Erythrocyte 3-0-methyl-D-glucose uptake assay for diagnosis of glucose-transporter-protein syndrome. J. Clin. Lab. Anal. 13, 116-121 (1999).

7 Tao, T., Tanizawa, Y., Matsutani, A., Matsubara, A., Kaneko, T. \& Kaku, K. HepG2/ erythrocyte glucose transporter (GLUT1) gene in NIDDM: a population association study and molecular scanning in Japanese subjects. Diabetologia. 38, 942-947 (1995).

8 Schouten, J. P., McElgunn, C. J., Waaijer, R., Zwijnenburg, D., Diepvens, F. \& Pals, G. Relative quantification of 40 nucleic acid sequences by multiplex ligation-dependent probe amplification. Nucleic. Acids. Res. 30, e57 (2002).

9 Wang, D., Pascual, J. M., Yang, H., Engelstad, K., Jhung, S., Sun, R. P. et al. Glut-1 deficiency syndrome: clinical, genetic, and therapeutic aspects. Ann. Neurol. 57, 111-118 (2005).

10 Klepper, J. \& Leiendecker, B. GLUT1 deficiency syndrome-2007 update. Dev. Med. Child. Neurol. 49, 707-716 (2007). 
11 Zorzi, G., Castellotti, B., Zibordi, F., Gellera, C. \& Nardocci, N. Paroxysmal movement disorders in GLUT1 deficiency syndrome. Neurology. 71, 146-148 (2008).

12 Rotstein, M., Engelstad, K., Yang, H., Wang, D., Levy, B., Chung, W. K. et al. Glut1 deficiency: inheritance pattern determined by haploinsufficiency. Ann. Neurol. 68, 955-958 (2010).

13 Mullen, S. A., Suls, A., De Jonghe, P., Berkovic, S. F. \& Scheffer, I. E. Absence epilepsies with widely variable onset are a key feature of familial GLUT1 deficiency. Neurology. 75, 432-440 (2010).

14 De Vivo, D. C. \& Wang, D. Glut1 deficiency: CSF glucose. How low is too low? Rev. Neurol. (Paris). 164, 877-880 (2008).

15 Wang, D., Yang, H., Shi, L., Ma, L., Fujii, T., Engelstad, K. et al. Functional studies of the T295M mutation causing Glut1 deficiency: glucose efflux preferentially affected by T295M. Pediatr. Res. 64, 538-543 (2008).
16 Leen, W. G., Klepper, J., Verbeek, M. M., Leferink, M., Hofste, T., van Engelen, B. G. et al. Glucose transporter-1 deficiency syndrome: the expanding clinical and genetic spectrum of a treatable disorder. Brain. 133, 655-670 (2010).

17 Haga, H., Yamada, R., Ohnishi, Y., Nakamura, Y. \& Tanaka, T. Gene-based SNP discovery as part of the Japanese Millennium Genome Project: identification of 190,562 genetic variations in the human genome. J. Hum. Genet. 47, 605-610 (2002).

18 Fujii, T., Morimoto, M., Yoshioka, H., Ho, Y. Y., Law, P. P., Wang, D. et al. T295Massociated Glut1 deficiency syndrome with normal erythrocyte 3-OMG uptake. Brain. Dev. 33, 316-320 (2011).

19 McAllister, M. S., Krizanac-Bengez, L., Macchia, F., Naftalin, R. J., Pedley, K. C., Mayberg, M. R. et al. Mechanisms of glucose transport at the blood-brain barrier: an in vitro study. Brain. Res. 409, 20-30 (2001). 